\title{
Anomalous top-Higgs couplings and top polarisation in single top and Higgs associated production at the LHC
}

\author{
Archil Kobakhidze, Lei Wu and Jason Yue \\ ARC Centre of Excellence for Particle Physics at the Terascale, \\ School of Physics, The University of Sydney, \\ NSW 2006, Australia \\ E-mail: archilk@physics.usyd.edu.au, leiwu@physics.usyd.edu.au, \\ j.yue@physics.usyd.edu.au
}

ABSTRACT: In this paper, we put constraints on anomalous $\mathcal{C P}$-violating top-Higgs couplings using the currently available Higgs data and explore the prospect of measuring these couplings at $240 \mathrm{GeV}$ TLEP. We find that the $\mathcal{C P}$-violating phase $\xi$ is currently limited in the range $|\xi|<0.6 \pi$ at $95 \%$ C.L. and may be further constrained to $|\xi|<0.07 \pi$ at TLEP. Under this consideration, we further investigate the observability of the scalar $(\xi=0)$, pseudoscalar $(\xi=0.5 \pi)$ and mixed $(\xi=0.25 \pi)$ top-Higgs interactions through the channel $p p \rightarrow t\left(\rightarrow \ell^{+} \nu_{\ell} b\right) h(\rightarrow b \bar{b}) j$. We find that it is most promising to observe pure pseudoscalar interactions with $y_{t}=y_{t}^{\mathrm{SM}}$, although this will be challenging due to a low signal to background ratio. We also find that the anomalous top-Higgs couplings can lead to sizeable differences in lepton forward-backward asymmetries and can be distinguished by measuring the lepton angular distributions from polarised top quarks at $14 \mathrm{TeV}$ LHC.

Keywords: Phenomenological Models, Hadronic Colliders

ARXIV EPRINT: 1406.1961 


\section{Contents}

1 Introduction 1

2 Higgs data constraints 2

3 Anomalous $t \bar{t} h$ couplings and $t h j$ production 3

$\begin{array}{lll}4 & \text { Conclusion } & 10\end{array}$

\section{Introduction}

The discovery of the Higgs boson at the Large Hadron Collider (LHC) $[1,2]$ is a major step towards elucidating the electroweak symmetry breaking (EWSB) mechanism. To ultimately establish its nature, a precise measurement of the Higgs couplings to fermions and gauge bosons, and Higgs self-coupling is an important task of experiments at LHC and future colliders [3-12]. Although the observed Higgs signals are still plagued with large uncertainties, they have at least firmly established the key Higgs production and decay channels predicted by the Standard Model (SM). In turn, the Higgs main production channel $g g \rightarrow h$ indirectly indicates the existence of top-Higgs interactions.

In the SM, the top quark is the heaviest fermion and hence has the strongest coupling to the Higgs boson. As such, it plays an important role in the EWSB and in various cosmological phenomena, such as electroweak phase transition and baryogenesis. The associated production of the top pair with Higgs boson have been widely investigated at the LHC [13-26] as a direct probe of the top-Higgs Yukawa coupling. Based on an integrated luminosity of $L=20.3 \mathrm{fb}^{-1}$, the ATLAS collaboration has analysed $p p \rightarrow t \bar{t} h$, with $h \rightarrow b \bar{b}$, and set a $95 \%$ C.L. limit [27] on the $t \bar{t} h$ cross section, $\sigma_{t \bar{t} h}<4.1 \sigma_{t \bar{t} h}^{\mathrm{SM}}$. The determination of the dominant background, $t \bar{t}+$ jets, is expected to be improved by the copious production of top quarks at the LHC [28]. However, even if the $t \bar{t} h$ coupling can be measured with sufficient accuracy, information on the relative phase between the top-Higgs Yukawa coupling and gauge-Higgs coupling will still be lacking.

In this regard, the search for Higgs boson production in association with a single top have proposed in refs. [29-32]. Like single top productions, there are three different production modes characterised by the virtuality of the $W$ boson [33]. The $t$ channel process $p p \rightarrow t h j$ with a space-like $W$ has the largest cross section amongst these production mode, reaching $\sim 88.2 \mathrm{fb}(14 \mathrm{TeV})$ at NLO QCD in the SM [34]. The most important feature of $t h j$ production is that the interference between the contributing processes with $h t \bar{t}$ and $h W W$ couplings allows direct examination both the modulus $\left(y_{t}\right)$ and the $\mathcal{C P}$ violating phase $(\xi)$ of the top-Higgs Yukawa coupling [33-38]. Such anomalous top-Higgs couplings may result from various new physics models [39-50]. The CMS collaboration has very recently 
presented the result on $t h j$ searches in the $h \rightarrow \gamma \gamma$ channel, and obtained a weak bound on the cross section of events with inverted top-Higgs coupling [51]. An equally important feature is that the top quark produced via the weak interaction in thj is left-handed in the SM. It is therefore expected that non-standard top-Higgs couplings will affect the polarisation states of top quark and change the angular distributions of the top quark decay products [36]. Such a polarisation asymmetry has been widely used to probe the anomalous top quark interactions at the LHC [52-69]. The precise measurement of thj channel opens a new window to probe the top quark Yukawa couplings and new physics at the LHC.

In this work, we examine the current and future constraints on the $\mathcal{C P}$-violating $t \bar{t} h$ couplings based on present LHC data and expected $240 \mathrm{GeV}$ TLEP sensitivity respectively. We investigate the observability of $p p \rightarrow t h j$ with $h \rightarrow b \bar{b}$ for the scalar, pseudoscalar and mixed interactions of top-Higgs. The potential to discriminate such anomalous top-Higgs coupling is studied by performing reconstructed-level Monte Carlo simulations at $14 \mathrm{TeV}$ HL-LHC. This paper is organised as follows: in section 2, we present the Higgs data constraints on the anomalous top quark Yukawa couplings; in section 3, we discuss their observability by analysing $p p \rightarrow t h j$ production; conclusions are drawn in section 4 .

\section{Higgs data constraints}

In some new physics models, the top quark Yukawa coupling can be different from the SM prediction. The new physics effects on $t \bar{t} h$ coupling can be parameterised by a minimal set of the gauge invariant dimension-six operators [39-44]. The most general Lagrangian of the $t \bar{t} h$ interaction in the broken phase can be parameterised as follows:

$$
\mathcal{L} \supset-\frac{y_{t}}{\sqrt{2}} \bar{t}\left(\cos \xi+i \gamma^{5} \sin \xi\right) t h, \quad \xi \in(-\pi, \pi]
$$

where $y_{t}$ takes the value $y_{t}^{\mathrm{SM}}=\sqrt{2} m_{t} / v$ and $\xi=0$ in the SM, with $v \approx 246 \mathrm{GeV}$ being the vacuum expectation value of the Higgs field. It is useful to define the scalar and pseudoscalar components of the anomalous top-Higgs interaction normalised to the treelevel SM coupling as $C_{S}=y_{t} \cos \xi / y_{t}^{\mathrm{SM}}$ and $C_{P}=y_{t} \sin \xi / y_{t}^{\mathrm{SM}}$ respectively. It should be noted that such $\mathcal{C P}$-violating interactions contribute to the electric dipole moment (EDM). However, the bounds on the coupling $C_{P}$ depend on the assumption of Higgs couplings to other light fermions [70, 71]. Since these couplings are practically unobservable at the LHC, we do not impose EDM constraints in this work. Other constraints from low-energy physics observables, such as $B_{s}-\bar{B}_{s}$ and $B \rightarrow X_{s} \gamma$, remain relatively weak [71].

An obvious consequence of non-standard top-Higgs interaction is that the production rate of $g g \rightarrow h$ and decay width of $h \rightarrow \gamma \gamma$ will deviate from those in the SM. In figure 1, we show the dependence of the reduced couplings $C_{h g g}$ and $C_{h \gamma \gamma}$ in terms of $y_{t}$ and $\xi$. It could be seen that the coupling $C_{h g g}$ at $\xi= \pm \pi / 2$ is larger than that at $\xi=0$. The reason is that the pseudoscalar interaction of $t \bar{t} h$ can lead to a larger form factor than the scalar interaction in the calculation of $g g \rightarrow h$. We can also notice that when $y_{t}$ increases, the reduced coupling $C_{h g g}$ becomes larger. However, this is different from the case in the coupling $C_{h \gamma \gamma}$, where the larger $y_{t}$ will induce a stronger cancellation between top quark loop and $W$ boson loop. 

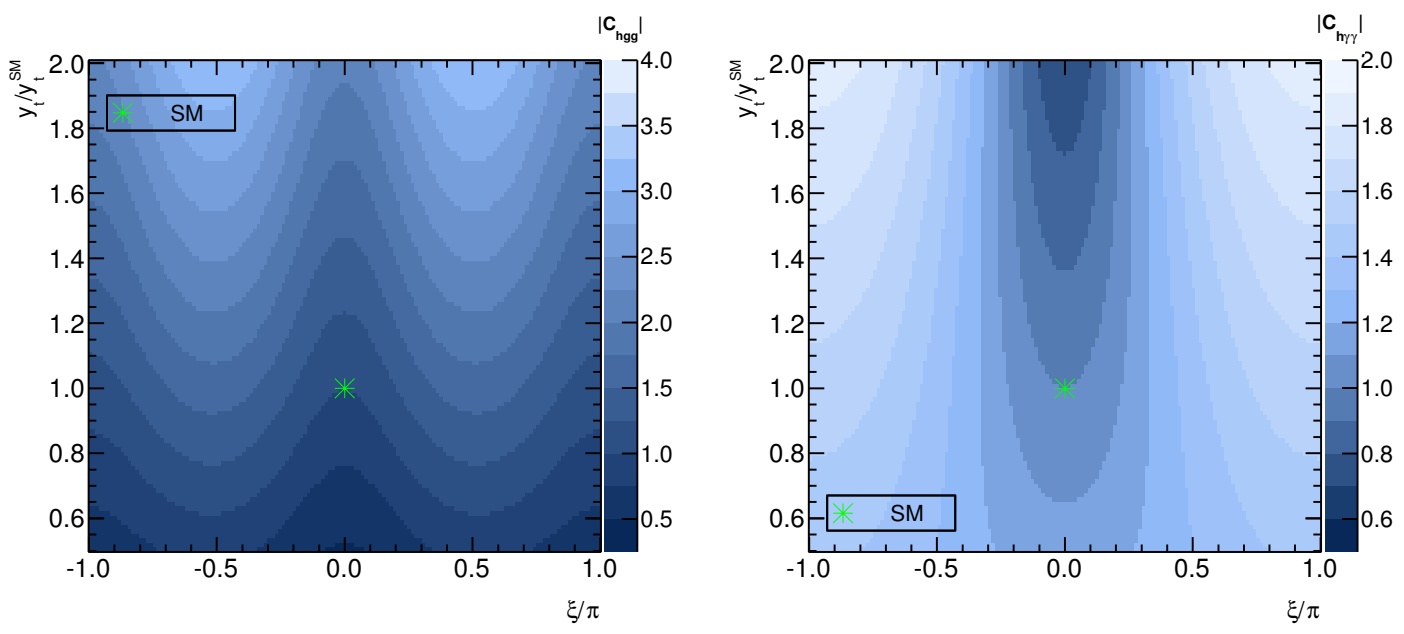

Figure 1. Reduced couplings $C_{h g g}$ and $C_{h \gamma \gamma}$ as a function of $y_{t}$ and $\xi$.

We impose the latest Higgs data constraints on the anomalous couplings $C_{S}$ and $C_{P}$ by calculating $\chi^{2}$ with the public package HiggsSignals-1.2.0 [72, 73], which includes all the available data sets from the ATLAS, CMS, CDF and DØ collaborations. In figure 2, we present the Higgs data constraints on the anomalous couplings $C_{S}$ and $C_{P}$ using current Higgs data and the prospect of improving the bounds at TLEP with $\sqrt{s}=240 \mathrm{GeV}$. We find that reduced pseudoscalar couplings in the range $\left|C_{P}\right|>0.6$ have been excluded at 95\% C.L by the current Higgs data and that positive scalar couplings $C_{S}>0.5$ are strongly favoured, consistent with refs. $[36,74,75]$. As the $\mathcal{C} \mathcal{P}$-phase $\xi$ increases from 0 to $\pi / 2$, the $95 \%$ C.L. allowed region for $y_{t} / y_{t}^{\mathrm{SM}}$ reduces from 0.7-1.2 to 0.4-0.6. The expected measurement of $C_{S}$ at $14 \mathrm{TeV}$ HL-LHC $\left(3000 \mathrm{fb}^{-1}\right)$ will further constrain $C_{P}$ to the range $\left|C_{P}\right|<0.4$. To estimate the bounds at TLEP, all measured Higgs couplings are assumed to be the same as the SM predictions, and the expected measurement uncertainties are taken from table 1-16 of ref. [76]. The main constraints come from the precise determinations of loop induced, reduced $C_{h g g}$ and $C_{h \gamma \gamma}$ couplings at TLEP. From figure 2, we see that the allowed range of $C_{P}$ at $95 \%$ C.L. shrinks to $\left|C_{P}\right|<0.2$, and $C_{S}$ is very close to one.

In figure 3 , we project the samples in the above $95 \%$ C.L. range allowed by the current Higgs data on the plane of the Higgs-diphoton reduced coupling $C_{h \gamma \gamma}$ versus the $\mathcal{C P}$ phase $\xi$. From figure 3 , we see that the effective coupling $C_{h \gamma \gamma}$ can be sizeably enhanced by the constructive contribution of the top-quark loop with $\mathcal{C P}$-violating couplings, reaching a maximal value of $\sim 1.32$. The bound on $\xi$ is expected to improve from $|\xi|<0.6 \pi$ at the LHC to $|\xi|<0.07 \pi$ at $240 \mathrm{GeV}$ TLEP.

\section{Anomalous $t \bar{t} h$ couplings and $t h j$ production}

In the numerical calculations we take the SM input parameters as follows [77]:

$$
\begin{aligned}
& m_{t}=173.07 \mathrm{GeV}, \quad m_{Z}=91.1876 \mathrm{GeV}, \quad \alpha\left(m_{Z}\right)=1 / 127.9, \\
& \sin ^{2} \theta_{W}=0.231, \quad m_{h}=125 \mathrm{GeV}, \quad \alpha_{s}\left(m_{Z}\right)=0.1185 .
\end{aligned}
$$



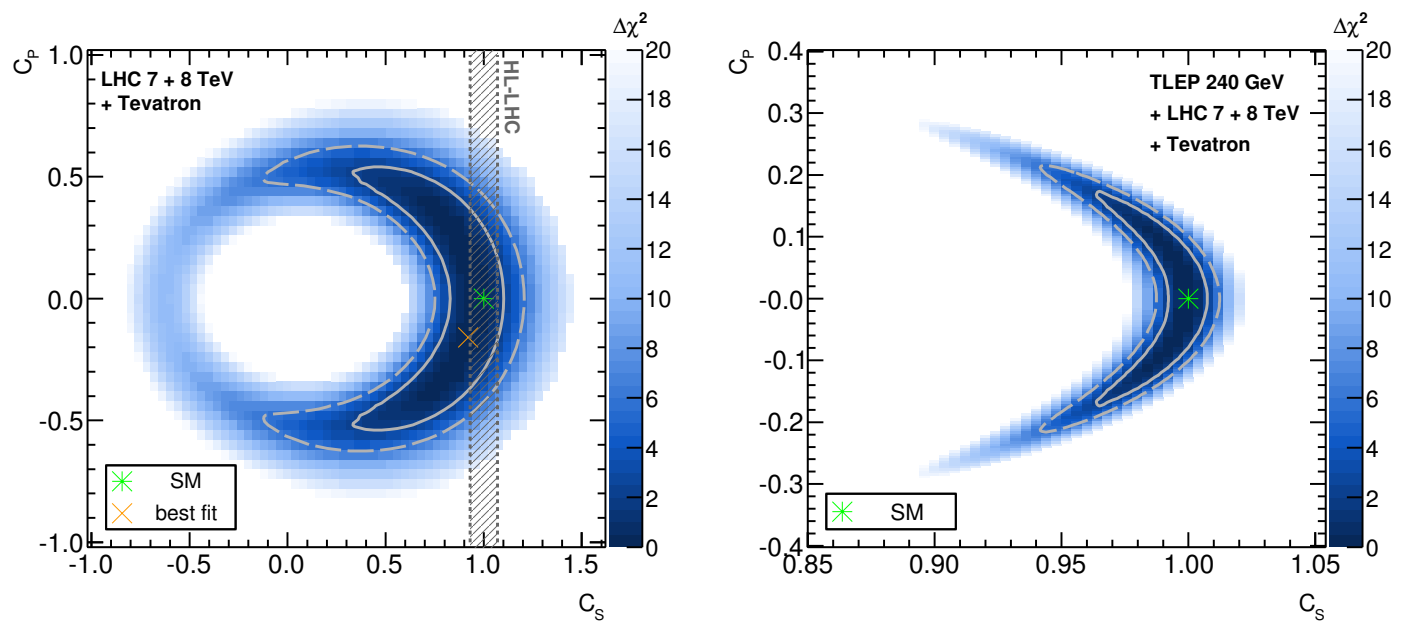

Figure 2. The Higgs data constraints on the anomalous couplings $C_{S}$ and $C_{P}$ at the LHC and the expected sensitivity of these couplings at $240 \mathrm{GeV}$ TLEP. The solid and dashed lines correspond to $68 \%$ and $95 \%$ C.L. respectively. The shadowed region represents the expected measurement uncertainty at HL-LHC.
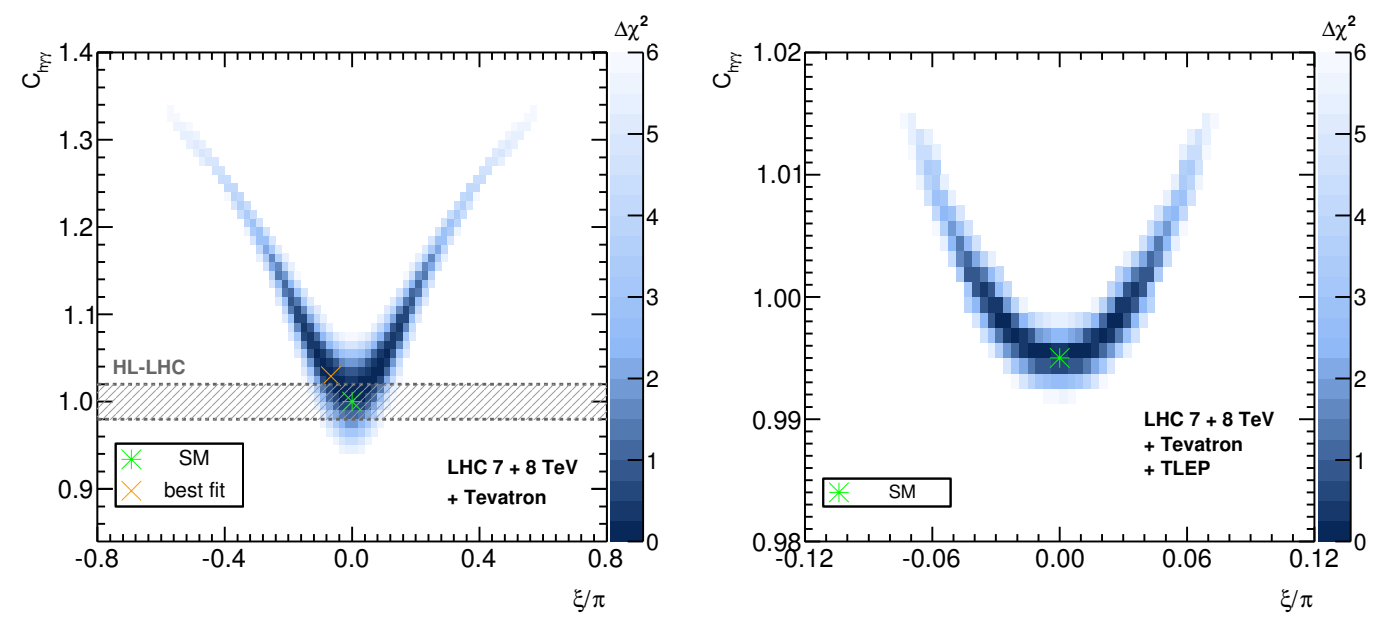

Figure 3. Left: the Higgs diphoton reduced coupling $C_{h \gamma \gamma}$ versus $\mathcal{C} \mathcal{P}$-violating phase $\xi$ in the $95 \%$ C.L allowed range in figure 2. The shaded region is the expected measurement uncertainty of $C_{h \gamma \gamma}$ at HL-LHC; right: the expected constraints on $C_{h \gamma \gamma}$ and $\xi$ at $240 \mathrm{GeV}$ TLEP.

By performing the Monte Carlo simulation, we investigate the observability of the anomalous top-Higgs couplings through the single top and Higgs associated production at the LHC

$$
p p \rightarrow t h j \rightarrow b \ell^{+} \nu b \bar{b} j,
$$

where $j$ denotes the light jets and $\ell^{+}=e^{+}, \mu^{+}$. Our signal is characterised by multi-jets ( 1 forward jet $+3 b$-jets) +1 lepton + missing energy (due to the neutrinos) in the final states. Although the $h \rightarrow b \bar{b}$ decay mode suffers a loss of efficiency in Higgs mass reconstruction, this shortcoming is mildly compensated by the large branching ratio of $h \rightarrow b \bar{b}$. Such a signature resembles the $t \bar{t} h$ topology analysed by ATLAS and CMS collaborations at the LHC Run-I, where at least $3 b$-jets are required [78, 79]. 
We implement the $\mathcal{C P}$-violating interaction of $t \bar{t} h$ in (2) by using the package FeynRules [80] and generate the parton-level signal and background events with MadGraph5 [81]. In figure 4 , we show the resulting the thj production cross section with no cuts on the final state kinematic distribution, as a function of $\xi$ for a selection of $y_{t}$ values. The values $y_{t}=0.7 y_{t}^{\mathrm{SM}}$ and $y_{t}=1.2 y_{t}^{\mathrm{SM}}$ respectively correspond to the $95 \%$ C.L. lower and upper bounds of $y_{t}$ for $\xi=0$, whilst $y_{t}=0.4 y_{t}^{\mathrm{SM}}$ is the lower bound for $\xi=\pi / 2$. We can see that the maximal value of the cross section occurs at $|\xi|=\pi$, due to the constructive interference of the contributions involving the $h W W$ and $h t \bar{t}$ couplings.

Parton showering and fast detector simulations are subsequently performed by PYTHIA [82] and Delphes [83]. Jets are clustered by using the anti- $k_{t}$ algorithm with a cone radius $\Delta R=0.7$ [84]. We keep the default cuts setting when generating the parton level events and set both the renormalisation $\left(\mu_{R}\right)$ and factorisation $\left(\mu_{F}\right)$ scale to the default event-by-event value and take CTEQ6L as the parton distribution function [85]. We adopt the $b$-jet tagging efficiency $\left(\epsilon_{b}\right)$ formula [87] that is a function of the transverse momentum and rapidity of the jets, with $\epsilon_{b}=0$ in the forward region $(|\eta|>2.5)$. We also include a misidentification probability of $10 \%$ and $1 \%$ for $c$-jets and light jets respectively. The mis-tag of QCD jets is assumed to be the default value as in Delphes. The number of events generated for both the signals and backgrounds in our calculations is $1.2 \times 10^{6}$.

The main SM backgrounds are: (B1) $p p \rightarrow t \bar{t}$, which can fake the signal when one light jet from the (anti-)top quark hadronic decay is misidentified as a $b$-jet. (B2) $p p \rightarrow t Z(\rightarrow b \bar{b}) j$, which is an irreducible background but with a pair of $b$-jets coming from $Z$ boson; and (B3) the irreducible QCD process $p p \rightarrow t b \bar{b} j$. Since the last two backgrounds have been demonstrated to be small [34, 38], we will focus on background (B1). To include the QCD effects, we generate parton-level events of $t \bar{t}$ with up to two jets that are matched to the parton shower using the MLM-scheme [86] with merging scale $x_{q}=20 \mathrm{GeV}$. We impose the basic cuts on the final states as follows:

$$
\begin{aligned}
\Delta R_{i j} & >0.4, & & i, j=b, j \text { or } \ell \\
p_{T}^{b} & >25 \mathrm{GeV}, & & \left|\eta_{b}\right|<2.5 \\
p_{T}^{\ell} & >25 \mathrm{GeV}, & & \left|\eta_{\ell}\right|<2.5 \\
p_{T}^{j} & >25 \mathrm{GeV}, & & \left|\eta_{j}\right|<4.7 .
\end{aligned}
$$

In figure 5, we plot the pseudorapidity distributions of the leading jet in the signals and backgrounds. It can be observed that most $t \bar{t}$ events of have a leading jet in the central region, which differs significantly from the signal, where a forward spectator jet accompanies the top quark and Higgs boson. The pseudorapidity of the leading jet is therefore required to satisfy $2.5<\left|\eta_{j_{1}}\right|<4.7$ in order to reduce the $t \bar{t}$ background.

Another cut which further suppresses the $t \bar{t}$ background is the invariant mass cut on the two $b$-jets from the Higgs boson decay. As proposed in ref. [38], the $b$-jet from the top quark decay can be tagged by selecting the minimal one among the invariant masses of each $b$-jet and the lepton, which should also satisfy $M_{b \ell}^{\min }<200 \mathrm{GeV}$. The remaining two $b$-jets are the considered as possible daughters of the Higgs boson for the signal. Given that the third $b$-jet in the $t \bar{t}$ background comes mainly from the misidentification of the jets in 


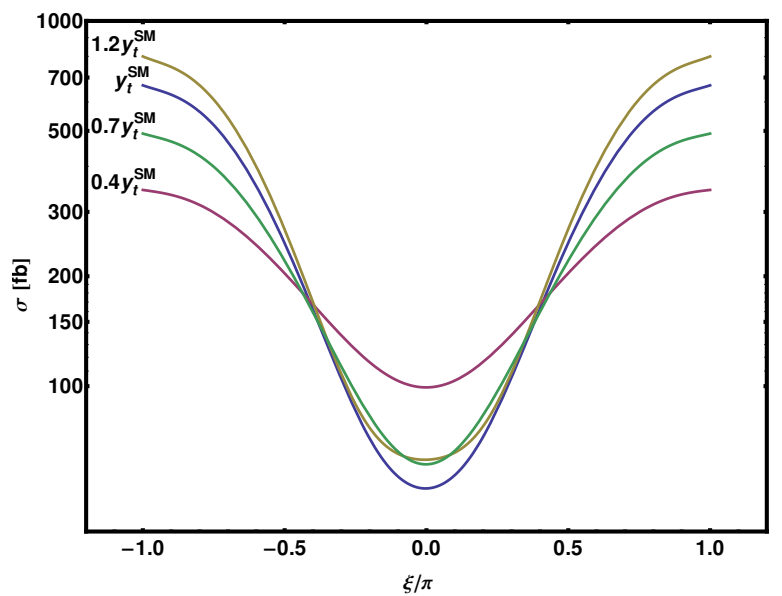

Figure 4. $p p \rightarrow t h j$ production cross section (fb) as a function of $\mathcal{C P}$ phase $\xi$ at $14 \mathrm{TeV}$ LHC. The conjugated process $\bar{t} h j$ is not included here.

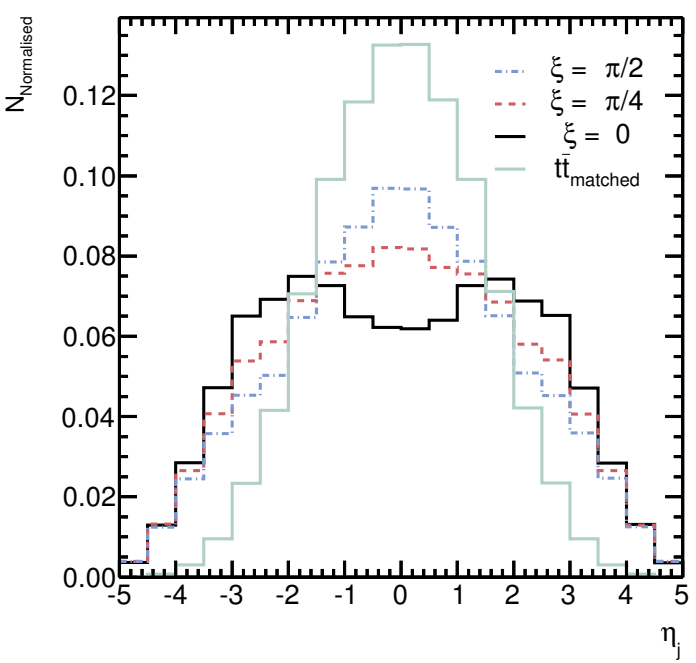

Figure 5. The pseudorapidity distributions of the leading jet in the signals and backgrounds.

the top quark hadronic decay, the invariant mass $M_{b \bar{b} j}^{\mathrm{rej}}$ and the two $b$-jets rejected by the minimisation of $M_{b \ell}$ should have a peak around $m_{t}$ for $t \bar{t}$, as is evident in figure 6 . We further find that the signal $M_{b \bar{b}}^{\mathrm{rej}}$ invariant mass peaks are more narrow than those of the backgrounds but are still relatively broad around the Higgs mass, reducing the effectiveness of the cut $\left|M_{b \bar{b}}^{\mathrm{rej}}-m_{h}\right|<15 \mathrm{GeV}$ in enhancing the observability of the signals.

Similar to figure 6 , we display $M_{b \bar{b} j}^{\mathrm{rej}}$ and $M_{b \bar{b}}^{\mathrm{rej}}$ distributions with a forward jet cut $2.5<\left|\eta_{j_{1}}\right|<4.7$ in figure 7 . Comparing with figure 6 , we can find that the peak of $M_{b \bar{b} j}^{\text {rej }}$ moves towards the high invariant mass region. This indicates that the selected forward jets in $t \bar{t}$ events are not from the top quark hadronic decay. We claim that the $M_{b \bar{b} j}^{\text {rej }}$ cut will not be very effective in improving the significance of the signal after the forward jet cut. The $M_{b \bar{b}}^{\text {rej }}$ distribution of the backgrounds becomes slightly more flat than the one in figure 6 . 

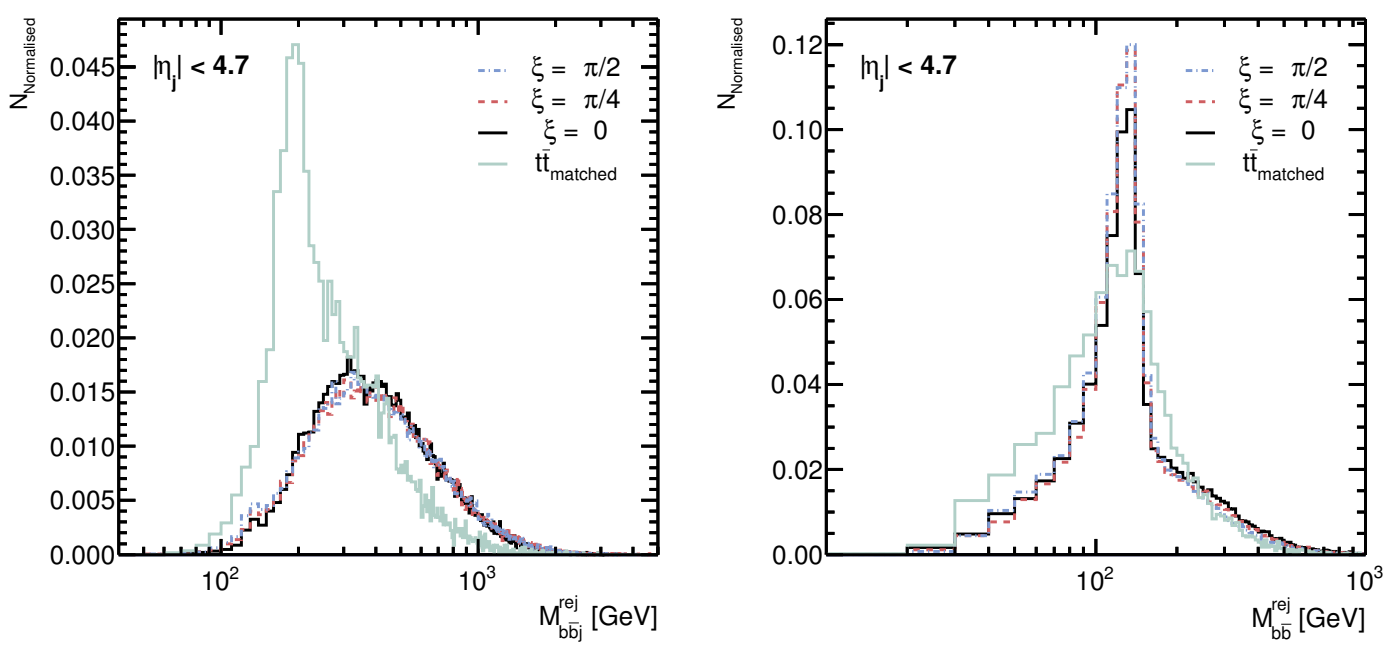

Figure 6. The normalised invariant mass distributions of $M_{b \bar{b}}^{r e c}$ and $M_{b \bar{b} j}^{r e c}$ with only basic cuts. Here the two $b$-jets are those rejected by the minimisation of $M_{b \ell}$.
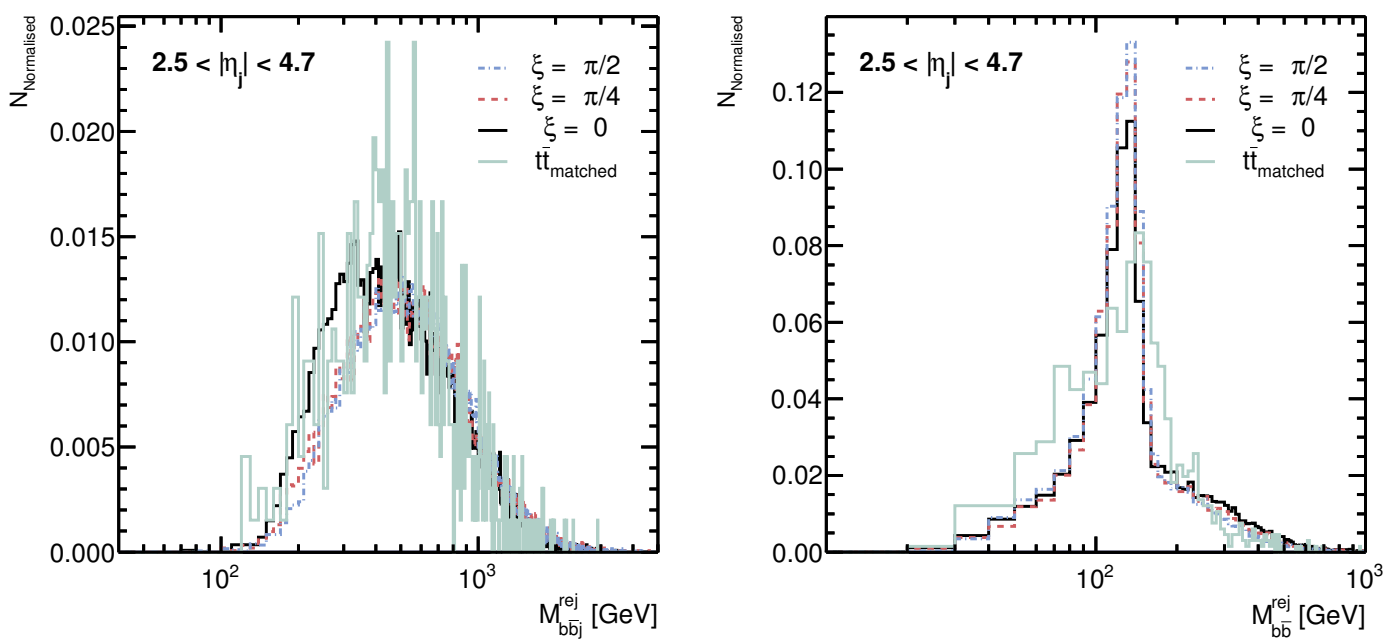

Figure 7. Same mass distributions as figure 6, but with an addition forward jet selection $2.5<\left|\eta_{j_{1}}\right|<4.7$.

In table 1, we summarise the cut-flow cross sections of the signal and backgrounds for $14 \mathrm{TeV}$ LHC. Considering that the $95 \%$ C.L. from the current LHC data still allows the $\mathcal{C P}$-violating phase $\xi$ to vary within $|\xi| \lesssim 0.6 \pi$ (cf. table 2 ), we take three benchmark points $\xi=0, \pi / 4$ and $\pi / 2$ and assume $y_{t}=y_{t}^{\mathrm{SM}}$ to demonstrate the observability of our signals. From table 1, we can find that: (1) since there is always a top quark in the signal and backgrounds, the identification procedure of $b$-jet from top quark using $M_{b \ell}^{\min }$ reduces both signal and background slightly; (2) the $t \bar{t}$ events can be further reduced by about one order with the forward jet selection, whilst the signal only loses about $1 / 3$ events; (3) the Higgs mass window cut can further remove about $3 / 4$ of the background events but keep almost $1 / 3$ of events of the signal. 


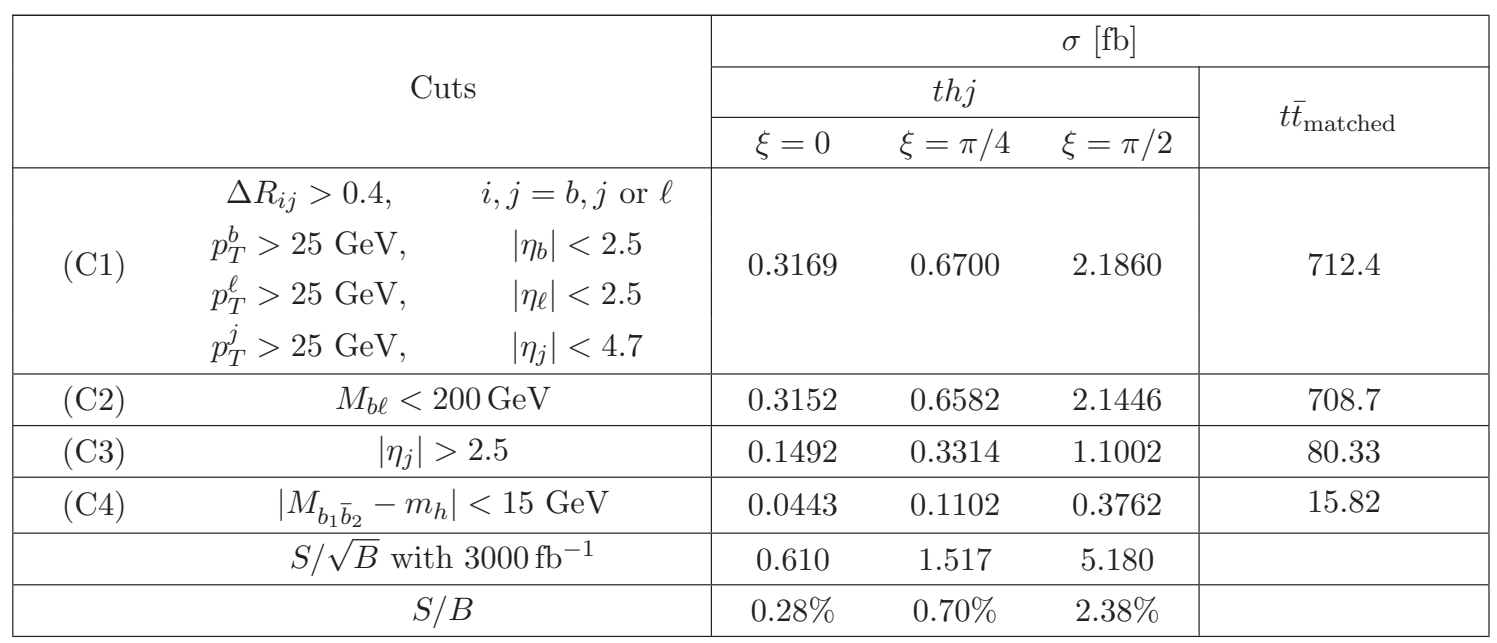

Table 1. Cutflow of the cross sections ( $\mathrm{fb}$ ) for the signals $(\xi=0, \pi / 4$ and $\pi / 2)$ and the backgrounds at $14 \mathrm{TeV}$ LHC. The conjugate process $p p \rightarrow \bar{t} h j$ has been included.

For each signal point, we calculate the statistical significance $S / \sqrt{B}$ and systematic significance $S / B$ for the luminosity $L=3000 \mathrm{fb}^{-1}$. From table 1 , we can see the observability of the pure pseudoscalar interaction $(\xi=\pi / 2)$ at $14 \mathrm{TeV}$ HL-LHC is the most promising, with $\mathrm{a} \sim 5 \sigma$ level statistical significance but a low systematic significance $\sim 2.4 \%$. Furthermore, it should be noted that when $\xi=\pi / 2$, the values of $y_{t} / y_{t}^{\mathrm{SM}}$ are required to be within the range $0.4-0.6$ to remain consistent with the current Higgs data. This will reduce the $t h j$ cross section of by a factor $\sim 2 / 3$, making the observation more challenging at the LHC.

Since the $\mathcal{C P}$-violating interaction described by 2 can affect the chirality of $t b W$ coupling through the interference between different Feynman diagrams, we will investigate the top quark polarisation asymmetry in the process $p p \rightarrow t\left(\rightarrow \ell^{+} \nu_{\ell} b\right) h(\rightarrow b \bar{b}) j$. The angular distribution of the lepton from a polarised top quark is given by:

$$
\frac{1}{\Gamma} \frac{d \Gamma}{d \cos \theta_{\ell}}=\frac{1}{2}\left(1+\mathcal{P}_{t} \kappa_{\ell} \cos \theta_{\ell}\right)
$$

where the lepton spin analysing power $\kappa_{\ell}$ is one at tree level in the SM, $\theta_{\ell}$ is the angle between the direction of the top quark and the lepton momenta in the rest frame of the top quark and $\mathcal{P}_{t}$ is the spin asymmetry. We reconstruct the top quark rest frame by minimising $\chi^{2}$, which is defined as:

$$
\chi^{2}=\left(\frac{m_{t}-m_{\ell \nu_{\ell} b}}{\Delta m_{t}}\right)^{2}
$$

where $\Delta m_{t}$ is taken as the SM top quark decay width [77]. With the on-shell condition of the $W$ boson and the top quark, the longitudinal momentum of the neutrino $p_{\nu L}$ can be determined as:

$$
p_{\nu L}=\frac{1}{2 p_{\ell T}^{2}}\left(A_{W} p_{\ell L} \pm E_{\ell} \sqrt{A_{W}^{2}-4 p_{\ell T}^{2} E_{T}^{2}}\right),
$$

where $A_{W}=m_{W}^{2}+2 \mathbf{p}_{\ell T} \cdot \mathbf{E}_{T}$. The ambiguity of the sign in (3.6) can be removed by the minimal $\chi^{2}$ requirement [88]. 

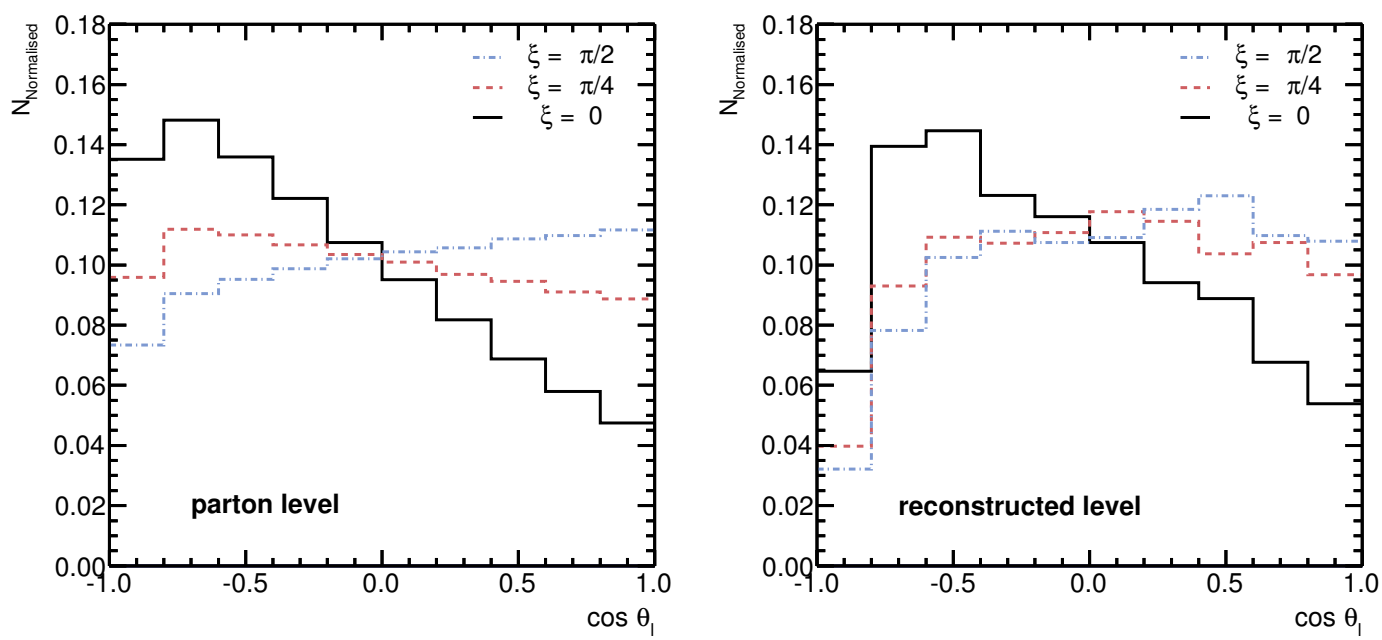

Figure 8. The angular distributions of the lepton from the top quark decay in the signal $p p \rightarrow t(\rightarrow$ $\left.\ell^{+} \nu_{\ell} b\right) h(\rightarrow b \bar{b}) j$ at parton level (left panel) and reconstruction level (right panel) after the cuts.

\begin{tabular}{|cccc|}
\hline$\xi$ & $\sigma(\cos \theta>0)[\mathrm{fb}]$ & $\sigma(\cos \theta<0)[\mathrm{fb}]$ & $\mathcal{A}_{\mathrm{FB}}^{\ell}(\%)$ \\
\hline 0 & 0.01458 & 0.0208 & -17.6 \\
\hline$\pi / 4$ & 0.04687 & 0.03991 & 8.0 \\
\hline$\pi / 2$ & 0.1681 & 0.1276 & 13.7 \\
\hline
\end{tabular}

Table 2. The reconstructed-level forward-backward asymmetry $\mathcal{A}_{\mathrm{FB}}^{\ell}$ at $14 \mathrm{TeV} \mathrm{LHC}$.

In figure 8, we plot the lepton angular distributions $\cos \theta_{\ell}$ for the $\mathcal{C P}$ phase $\xi=0, \pi / 4$ and $\pi / 2$ in the signal $p p \rightarrow t\left(\rightarrow \ell^{+} \nu_{\ell} b\right) h(\rightarrow b \bar{b}) j$ at parton level and reconstruction level respectively after the cuts (C3) and (C4) (cf. table 1). From figure 8 , we can see that the direction of the lepton is inclined to be opposite to its parent top quark for $\xi=0$, which corresponds to the SM top-Higgs interaction. However, the mixed and pseudoscalar interactions will affect the polarisation state of the top quark and change the slopes such that the number of events with $\cos \theta_{\ell}>0$ is larger than those with $\cos \theta_{\ell}<0$. The differences between the slopes are diluted from the parton level to the reconstruction level.

Based on the angular distributions, we can further define the lepton forward-backward (FB) asymmetry:

$$
\mathcal{A}_{\mathrm{FB}}^{\ell}=\frac{\sigma\left(\cos \theta_{\ell}>0\right)-\sigma\left(\cos \theta_{\ell}<0\right)}{\sigma\left(\cos \theta_{\ell}>0\right)+\sigma\left(\cos \theta_{\ell}<0\right)} .
$$

In table 2 , we present the values of $A_{\mathrm{FB}}^{\ell}$ for $\xi=0, \pi / 4$ and $\pi / 2$ in the signal $p p \rightarrow$ $t\left(\rightarrow \ell^{+} \nu_{\ell} b\right) h(\rightarrow b \bar{b}) j$ at $14 \mathrm{TeV}$ LHC. From table 2 , we can see that both of the scalar and pseudoscalar top-Higgs interaction can induce large forward-backward asymmetries but with different signs, reaching $-17.6 \%$ and $13.7 \%$ respectively. The measurement of the lepton forward-backward asymmetry can distinguish the scalar and pseudoscalar top-Higgs interactions at the LHC. 


\section{Conclusion}

In this paper, we have obtained constraints on the $\mathcal{C P}$-violating top-Higgs couplings using the current Higgs data and found that values of $\mathcal{C} \mathcal{P}$-violating phase $|\xi|>0.6 \pi$ are already excluded at $95 \%$ C.L.. We expected TLEP to improve this exclusion region to $|\xi|>0.07 \pi$. With current constraints on $\xi$, we further investigate the observability of the scalar, pseudoscalar and mixed top-Higgs interactions through the channel $p p \rightarrow t\left(\rightarrow \ell^{+} \nu_{\ell} b\right) h(\rightarrow b \bar{b}) j$. We found that it is most promising to observe pure pseudoscalar interactions at the HLLHC but it is still challenging due to a low $S / B$ ratio. However, the anomalous top-Higgs couplings can lead to sizeable differences in forward-backward asymmetries and can be distinguished by measuring the lepton angular distributions from polarised top quarks at $14 \mathrm{TeV}$ LHC.

\section{Acknowledgments}

This work was supported by the Australian Research Council. Lei Wu is also supported by the National Natural Science Foundation of China (NNSFC) under grants Nos. 11222548, 11275057 and 11305049, by Specialised Research Fund for the Doctoral Program of Higher Education under Grant No. 20134104120002.

Open Access. This article is distributed under the terms of the Creative Commons Attribution License (CC-BY 4.0), which permits any use, distribution and reproduction in any medium, provided the original author(s) and source are credited.

\section{References}

[1] ATLAS collaboration, Combined search for the Standard Model Higgs boson using up to $4.9 \mathrm{fb}^{-1}$ of pp collision data at $\sqrt{s}=7 \mathrm{TeV}$ with the ATLAS detector at the LHC, Phys. Lett. B 710 (2012) 49 [arXiv:1202.1408] [INSPIRE].

[2] CMS collaboration, Combined results of searches for the standard model Higgs boson in pp collisions at $\sqrt{s}=7 \mathrm{TeV}$, Phys. Lett. B 710 (2012) 26 [arXiv:1202.1488] [INSPIRE].

[3] S. Dawson et al., Working Group Report: Higgs Boson, arXiv:1310.8361 [INSPIRE].

[4] C. Englert et al., Precision Measurements of Higgs Couplings: Implications for New Physics Scales, J. Phys. G 41 (2014) 113001 [arXiv:1403.7191] [INSPIRE].

[5] V. Barger, L.L. Everett, H.E. Logan and G. Shaughnessy, Scrutinizing the 125 GeV Higgs boson in two Higgs doublet models at the LHC, ILC and Muon Collider, Phys. Rev. D 88 (2013) 115003 [arXiv:1308.0052] [InSPIRE].

[6] A. Kobakhidze and J. Yue, Excluding a Generic Spin-2 Higgs Impostor, Phys. Lett. B 727 (2013) 456 [arXiv:1310.0151] [INSPIRE].

[7] N. Liu, J. Ren, L. Wu, P. Wu and J.M. Yang, Full one-loop electroweak corrections to $e^{+} e^{-} \rightarrow Z H \gamma$ at a Higgs factory, JHEP 04 (2014) 189 [arXiv:1311.6971] [INSPIRE].

[8] Z. Heng, L. Shang and P. Wan, Pair production of a $125 \mathrm{GeV}$ Higgs boson in MSSM and NMSSM at the ILC, JHEP 10 (2013) 047 [arXiv:1306.0279] [INSPIRE]. 
[9] S.L. Hu, N. Liu, J. Ren and L. Wu, Revisiting Associated Production of $125 \mathrm{GeV}$ Higgs Boson with a Photon at a Higgs Factory, arXiv:1402.3050 [INSPIRE].

[10] L. Wang and X.-F. Han, Study of the heavy CP-even Higgs with mass $125 \mathrm{GeV}$ in two-Higgs-doublet models at the LHC and ILC, arXiv:1404.7437 [INSPIRE].

[11] C. Han, A. Kobakhidze, N. Liu, L. Wu and B. Yang, Constraining Top partner and Naturalness at the LHC and TLEP, arXiv:1405.1498 [INSPIRE].

[12] J. Cao, Z. Heng, D. Li, L. Shang and P. Wu, Higgs-strahlung production process $e^{+} e^{-} \rightarrow Z h$ at the future Higgs factory in the Minimal Dilaton Model, JHEP 08 (2014) 138 [arXiv: 1405.4489] [INSPIRE].

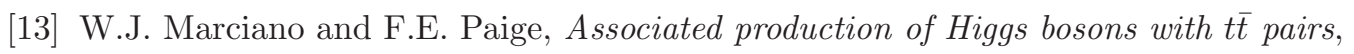
Phys. Rev. Lett. 66 (1991) 2433 [INSPIRE].

[14] J. Dai, J.F. Gunion and R. Vega, Using $b$ tagging to detect tt-Higgs-boson production with $H \rightarrow b \bar{b}$, Phys. Rev. Lett. 71 (1993) 2699 [hep-ph/9306271] [INSPIRE].

[15] J. Goldstein, C.S. Hill, J. Incandela, S.J. Parke, D.L. Rainwater and D. Stuart, $p \bar{p} \rightarrow t \bar{t} H: a$ discovery mode for the Higgs boson at the Tevatron, Phys. Rev. Lett. 86 (2001) 1694 [hep-ph/0006311] [INSPIRE].

[16] A. Belyaev and L. Reina, $p p \rightarrow t \bar{t} H, H \rightarrow \tau^{+} \tau^{-}$: Toward a model independent determination of the Higgs boson couplings at the LHC, JHEP 08 (2002) 041 [hep-ph/0205270] [INSPIRE].

[17] F. Maltoni, D.L. Rainwater and S. Willenbrock, Measuring the top quark Yukawa coupling at hadron colliders via $t \bar{t} H, H \rightarrow W^{+} W^{-}$, Phys. Rev. D 66 (2002) 034022 [hep-ph/0202205] [INSPIRE].

[18] V. Drollinger, T. Müller and D. Denegri, Searching for Higgs bosons in association with top quark pairs in the $H^{0} \rightarrow b \bar{b}$ decay mode, hep-ph/0111312 [INSPIRE].

[19] W. Beenakker, S. Dittmaier, M. Krämer, B. Plumper, M. Spira and P.M. Zerwas, NLO QCD corrections to $t \bar{t} H$ production in hadron collisions, Nucl. Phys. B 653 (2003) 151 [hep-ph/0211352] [INSPIRE].

[20] P. Agrawal, S. Bandyopadhyay and S.P. Das, Multilepton Signatures of the Higgs Boson through its Production in Association with a Top-quark Pair, Phys. Rev. D 88 (2013) 093008 [arXiv: 1308.3043] [INSPIRE].

[21] S. Biswas, R. Frederix, E. Gabrielli and B. Mele, Enhancing the $t \bar{t} H$ signal through top-quark spin polarization effects at the LHC, JHEP 07 (2014) 020 [arXiv: 1403.1790] [INSPIRE].

[22] M.V. Garzelli, A. Kardos, C.G. Papadopoulos and Z. Trócsányi, Standard Model Higgs boson production in association with a top anti-top pair at NLO with parton showering, Europhys. Lett. 96 (2011) 11001 [arXiv:1108.0387] [INSPIRE].

[23] R. Frederix, S. Frixione, V. Hirschi, F. Maltoni, R. Pittau and P. Torrielli, Scalar and pseudoscalar Higgs production in association with a top-antitop pair, Phys. Lett. B 701 (2011) 427 [arXiv:1104.5613] [INSPIRE].

[24] C. Degrande, J.M. Gerard, C. Grojean, F. Maltoni and G. Servant, Probing Top-Higgs Non-Standard Interactions at the LHC, JHEP 07 (2012) 036 [Erratum ibid. 1303 (2013) 032] [arXiv: 1205.1065] [INSPIRE].

[25] D. Curtin, J. Galloway and J.G. Wacker, Measuring the tīh coupling from same-sign dilepton +2b measurements, Phys. Rev. D 88 (2013) 093006 [arXiv:1306.5695] [INSPIRE]. 
[26] J. Adelman, A. Loginov, P. Tipton and J. Vasquez, Study of $t \bar{t} H\left(H \rightarrow \mu^{+} \bar{\mu}^{-}\right)$in the three lepton channel at $\sqrt{s}=14 \mathrm{TeV}$; a Snowmass white paper, arXiv:1310.1132 [INSPIRE].

[27] ATLAS collaboration, Search for the Standard Model Higgs boson produced in association with top quarks and decaying to $b \bar{b}$ in pp collisions at $\sqrt{s}=8 \mathrm{TeV}$ with the ATLAS detector at the LHC, ATLAS-CONF-2014-011 (2014).

[28] CMS collaboration, First measurement of the cross section ratio $\sigma(t \bar{t} b \bar{b}) / \sigma(t \bar{t} j j)$ in $p p$ collisions at $\sqrt{s}=7 \mathrm{GeV}$, CMS-PAS-TOP-12-024.

[29] G. Bordes and B. van Eijk, On the associate production of a neutral intermediate mass Higgs boson with a single top quark at the LHC and SSC, Phys. Lett. B 299 (1993) 315 [INSPIRE].

[30] A. Ballestrero and E. Maina, $t \bar{b} H$ production for an intermediate mass Higgs, Phys. Lett. B 299 (1993) 312 [inSPIRE].

[31] W.J. Stirling and D.J. Summers, Production of an intermediate mass Higgs boson in association with a single top quark at LHC and SSC, Phys. Lett. B 283 (1992) 411 [INSPIRE].

[32] J.L. Diaz-Cruz and O.A. Sampayo, Associated production of the Higgs boson with $t \bar{b}$ at hadron colliders, Phys. Lett. B 276 (1992) 211 [INSPIRE].

[33] F. Maltoni, K. Paul, T. Stelzer and S. Willenbrock, Associated production of Higgs and single top at hadron colliders, Phys. Rev. D 64 (2001) 094023 [hep-ph/0106293] [INSPIRE].

[34] M. Farina, C. Grojean, F. Maltoni, E. Salvioni and A. Thamm, Lifting degeneracies in Higgs couplings using single top production in association with a Higgs boson, JHEP 05 (2013) 022 [arXiv:1211.3736] [INSPIRE].

[35] S. Biswas, E. Gabrielli and B. Mele, Single top and Higgs associated production as a probe of the Htt coupling sign at the LHC, JHEP 01 (2013) 088 [arXiv:1211.0499] [INSPIRE].

[36] J. Ellis, D.S. Hwang, K. Sakurai and M. Takeuchi, Disentangling Higgs-Top Couplings in Associated Production, JHEP 04 (2014) 004 [arXiv: 1312.5736] [INSPIRE].

[37] C. Englert and E. Re, Bounding the top Yukawa with Higgs-associated single-top production, Phys. Rev. D 89 (2014) 073020 [arXiv: 1402.0445] [InSPIRE].

[38] J. Chang, K. Cheung, J.S. Lee and C.-T. Lu, Probing the Top-Yukawa Coupling in Associated Higgs production with a Single Top Quark, JHEP 05 (2014) 062 [arXiv: 1403.2053] [INSPIRE].

[39] J.A. Aguilar-Saavedra, A minimal set of top-Higgs anomalous couplings, Nucl. Phys. B 821 (2009) 215 [arXiv:0904.2387] [INSPIRE].

[40] C. Zhang, N. Greiner and S. Willenbrock, Constraints on Non-standard Top Quark Couplings, Phys. Rev. D 86 (2012) 014024 [arXiv:1201.6670] [INSPIRE].

[41] X. Zhang, S.K. Lee, K. Whisnant and B.L. Young, Phenomenology of a nonstandard top quark Yukawa coupling, Phys. Rev. D 50 (1994) 7042 [hep-ph/9407259] [INSPIRE].

[42] P. Agrawal, S. Mitra and A. Shivaji, Effect of Anomalous Couplings on the Associated Production of a Single Top Quark and a Higgs Boson at the LHC, JHEP 12 (2013) 077 [arXiv: 1211.4362] [INSPIRE].

[43] Y. Wang, F.P. Huang, C.S. Li, B.H. Li, D.Y. Shao and J. Wang, Constraints on flavor-changing neutral-current Htq couplings from the signal of $t H$ associated production with QCD next-to-leading order accuracy at the LHC, Phys. Rev. D 86 (2012) 094014 [arXiv: 1208.2902] [INSPIRE]. 
[44] G. Bhattacharyya, D. Das and P.B. Pal, Modified Higgs couplings and unitarity violation, Phys. Rev. D 87 (2013) 011702 [arXiv:1212.4651] [InSPIRE].

[45] A. Djouadi, The anatomy of electro-weak symmetry breaking. II. The Higgs bosons in the minimal supersymmetric model, Phys. Rept. 459 (2008) 1 [hep-ph/0503173] [INSPIRE].

[46] A. Djouadi and G. Moreau, The couplings of the Higgs boson and its CP properties from fits of the signal strengths and their ratios at the $7+8 \mathrm{TeV} L H C$,

Eur. Phys. J. C 73 (2013) 2512 [arXiv:1303.6591] [INSPIRE].

[47] D. McKeen, M. Pospelov and A. Ritz, Modified Higgs branching ratios versus CP and lepton flavor violation, Phys. Rev. D 86 (2012) 113004 [arXiv:1208.4597] [INSPIRE].

[48] A. Celis, V. Ilisie and A. Pich, LHC constraints on two-Higgs doublet models, JHEP 07 (2013) 053 [arXiv:1302.4022] [InSPIRE].

[49] J. Shu and Y. Zhang, Impact of a CP-violating Higgs Sector: From LHC to Baryogenesis, Phys. Rev. Lett. 111 (2013) 091801 [arXiv:1304.0773] [INSPIRE].

[50] A. Kobakhidze, Standard Model with a distorted Higgs sector and the enhanced Higgs diphoton decay rate, arXiv: 1208.5180 [INSPIRE].

[51] CMS collaboration, Search for associated production of a single top quark and a Higgs boson in events where the Higgs boson decays to two photons at $\sqrt{s}=8 \mathrm{TeV}$, CMS-PAS-HIG-14-001.

[52] A. Prasath, R.M. Godbole and S.D. Rindani, Top polarisation measurement and anomalous Wtb coupling, arXiv:1405.1264 [INSPIRE].

[53] J.A. Aguilar-Saavedra and S.A. dos Santos, New directions for top quark polarization in the t-channel process, Phys. Rev. D 89 (2014) 114009 [arXiv:1404.1585] [InSPIRE].

[54] S. Biswas, R. Frederix, E. Gabrielli and B. Mele, Enhancing the $t \bar{t} H$ signal through top-quark spin polarization effects at the LHC, JHEP 07 (2014) 020 [arXiv:1403.1790] [INSPIRE].

[55] W. Bernreuther and Z.-G. Si, Top quark spin correlations and polarization at the LHC: standard model predictions and effects of anomalous top chromo moments, Phys. Lett. B 725 (2013) 115 [arXiv: 1305. 2066] [INSPIRE].

[56] Q.-H. Cao, X. Wan, X.-p. Wang and S.-h. Zhu, Searching for Charged Higgs Boson in Polarized Top Quark, Phys. Rev. D 87 (2013) 055022 [arXiv:1301.6608] [InSPIRE].

[57] B. Yang and N. Liu, One-loop effects on top pair production in the littlest Higgs model with T-parity at the LHC, Eur. Phys. J. C 73 (2013) 2570 [arXiv:1210.5120] [InSPIRE].

[58] J.A. Aguilar-Saavedra and R.V. Herrero-Hahn, Model-independent measurement of the top quark polarisation, Phys. Lett. B 718 (2013) 983 [arXiv:1208.6006] [INSPIRE].

[59] X.-Q. Li, Z.-G. Si, K. Wang, L. Wang, L. Zhang and G. Zhu, Light Top Squark in Precision Top Quark Sample, Phys. Rev. D 89 (2014) 077703 [arXiv:1311.6874] [InSPIRE].

[60] I. Low, Polarized charginos (and top quarks) in scalar top quark decays, Phys. Rev. D 88 (2013) 095018 [arXiv:1304.0491] [InSPIRE].

[61] R.M. Godbole, L. Hartgring, I. Niessen and C.D. White, Top polarisation studies in $H^{-} t$ and Wt production, JHEP 01 (2012) 011 [arXiv:1111.0759] [INSPIRE].

[62] J. Cao, K. Hikasa, L. Wang, L. Wu and J.M. Yang, Testing new physics models by top charge asymmetry and polarization at the LHC, Phys. Rev. D 85 (2012) 014025 [arXiv:1109.6543] [INSPIRE]. 
[63] D. Krohn, T. Liu, J. Shelton and L.-T. Wang, A polarized view of the top asymmetry, Phys. Rev. D 84 (2011) 074034 [arXiv: 1105.3743] [InSPIRE].

[64] J. Cao, L. Wu and J.M. Yang, New physics effects on top quark spin correlation and polarization at the LHC: a comparative study in different models, Phys. Rev. D 83 (2011) 034024 [arXiv:1011.5564] [INSPIRE].

[65] D. Choudhury, R.M. Godbole, S.D. Rindani and P. Saha, Top polarization, forward-backward asymmetry and new physics, Phys. Rev. D 84 (2011) 014023 [arXiv: 1012.4750] [InSPIRE].

[66] D.-W. Jung, P. Ko and J.S. Lee, Longitudinal top polarization as a probe of a possible origin of forward-backward asymmetry of the top quark at the Tevatron, Phys. Lett. B 701 (2011) 248 [arXiv:1011.5976] [INSPIRE].

[67] W. Bernreuther and Z.-G. Si, Distributions and correlations for top quark pair production and decay at the Tevatron and LHC., Nucl. Phys. B 837 (2010) 90 [arXiv:1003.3926] [INSPIRE].

[68] R. Schwienhorst, C.-P. Yuan, C. Mueller and Q.-H. Cao, Single top quark production and decay in the t-channel at next-to-leading order at the LHC, Phys. Rev. D 83 (2011) 034019 [arXiv: 1012.5132] [INSPIRE].

[69] J. Shelton, Polarized tops from new physics: signals and observables, Phys. Rev. D 79 (2009) 014032 [arXiv: 0811.0569] [inSPIRE].

[70] D. Atwood, S. Bar-Shalom, G. Eilam and A. Soni, CP violation in top physics, Phys. Rept. 347 (2001) 1 [hep-ph/0006032] [INSPIRE].

[71] J. Brod, U. Haisch and J. Zupan, Constraints on CP-violating Higgs couplings to the third generation, JHEP 11 (2013) 180 [arXiv:1310.1385] [INSPIRE].

[72] P. Bechtle, S. Heinemeyer, O. Stål, T. Stefaniak and G. Weiglein, HiggsSignals: Confronting arbitrary Higgs sectors with measurements at the Tevatron and the LHC,

Eur. Phys. J. C 74 (2014) 2711 [arXiv:1305.1933] [INSPIRE].

[73] P. Bechtle, O. Brein, S. Heinemeyer, G. Weiglein and K.E. Williams, HiggsBounds: Confronting Arbitrary Higgs Sectors with Exclusion Bounds from LEP and the Tevatron, Comput. Phys. Commun. 181 (2010) 138 [arXiv:0811.4169] [inSPIRE].

[74] H. Belusca-Maito, Effective Higgs Lagrangian and Constraints on Higgs Couplings, arXiv: 1404.5343 [INSPIRE].

[75] K. Nishiwaki, S. Niyogi and A. Shivaji, ttH Anomalous Coupling in Double Higgs Production, JHEP 04 (2014) 011 [arXiv:1309.6907] [INSPIRE].

[76] S. Dawson et al., Working Group Report: Higgs Boson, arXiv:1310.8361 [INSPIRE].

[77] Particle Data Group collaboration, J. Beringer et al., Review of Particle Physics (RPP), Phys. Rev. D 86 (2012) 010001 [inSPIRE].

[78] ATLAS collaboration, Search for the Standard Model Higgs boson produced in association with top quarks in proton-proton collisions at $\sqrt{s}=7 \mathrm{TeV}$ using the ATLAS detector, ATLAS-CONF-2012-135 (2012).

[79] CMS collaboration, Search for Higgs boson production in association with top quark pairs in pp collisions, CMS-PAS-HIG-12-025.

[80] A. Alloul, N.D. Christensen, C. Degrande, C. Duhr and B. Fuks, FeynRules 2.0 - a complete toolbox for tree-level phenomenology, Comput. Phys. Commun. 185 (2014) 2250 [arXiv: 1310.1921] [INSPIRE]. 
[81] J. Alwall, M. Herquet, F. Maltoni, O. Mattelaer and T. Stelzer, MadGraph 5: Going Beyond, JHEP 06 (2011) 128 [arXiv:1106.0522] [INSPIRE].

[82] T. Sjöstrand, S. Mrenna and P.Z. Skands, PYTHIA 6.4 Physics and Manual, JHEP 05 (2006) 026 [hep-ph/0603175] [INSPIRE].

[83] DELPHES 3 collaboration, J. de Favereau et al., DELPHES 3, a modular framework for fast simulation of a generic collider experiment, JHEP 02 (2014) 057 [arXiv:1307.6346] [INSPIRE].

[84] M. Cacciari, G.P. Salam and G. Soyez, The anti-kt jet clustering algorithm, JHEP 04 (2008) 063 [arXiv: 0802.1189] [InSPIRE].

[85] J. Pumplin, A. Belyaev, J. Huston, D. Stump and W.K. Tung, Parton distributions and the strong coupling: CTEQ6AB PDFs, JHEP 02 (2006) 032 [hep-ph/0512167] [INSPIRE].

[86] M.L. Mangano, M. Moretti, F. Piccinini and M. Treccani, Matching matrix elements and shower evolution for top-quark production in hadronic collisions, JHEP 01 (2007) 013 [hep-ph/0611129] [INSPIRE].

[87] CMS collaboration, b-Jet Identification in the CMS Experiment, CMS-PAS-BTV-11-004.

[88] E.L. Berger, Q.-H. Cao, J.-H. Yu and C.-P. Yuan, Calculation of Associated Production of a Top Quark and $a W^{\prime}$ at the LHC, Phys. Rev. D 84 (2011) 095026 [arXiv:1108.3613] [INSPIRE]. 